\title{
Ampicillin Sodium
}

National Cancer Institute

\section{Source}

National Cancer Institute. Ampicillin Sodium. NCI Thesaurus. Code C47959.

The sodium salt form of ampicillin, a broad-spectrum semisynthetic derivative of aminopenicillin. Ampicillin sodium inhibits bacterial cell wall synthesis by binding to penicillin binding proteins, thereby inhibiting peptidoglycan synthesis, a critical component of the bacterial cell wall. 